\title{
A MINIMAL MODEL FOR SET THEORY
}

\author{
BY PAUL J. COHEN ${ }^{1}$
}

Communicated by Paul R. Halmos, March 22, 1963

In the proof of the consistency of the Continuum Hypothesis and the Axiom of Choice with the other axioms of set theory, Gödel [1] introduced the notion of a constructible set and showed that the constructible sets form a model for set theory. These sets are intuitively those which can be reached by means of a transfinite sequence of several simple operations. He then showed that the Axiom of Choice and Continuum Hypothesis held in the collection of constructible sets. If the original universe of sets is sufficiently rich in ordinal numbers, it will follow that every set is constructible, in which case we say that the Axiom of Constructibility is satisfied. This axiom implies the two axioms previously mentioned. However, from one point of view it may seem that this notion of constructibility does not intuitively correspond to what is meant by constructive since it may happen that all sets in the universe are constructive. In this paper we show that a more restricted notion of "construction" will yield a class of sets which form a minimal model for set theory. In this manner we prove the consistency of a stronger form of the Axiom of Constructibility. We observe that the idea of a minimal collection of objects satisfying certain axioms is well known in mathematics, for example, in group theory one often considers the subgroup generated by a collection of elements, and in measure theory we define the Borel sets as the smallest $\sigma$-algebra of sets containing the open sets.

We shall work within the framework of Zermelo-Frankel set theory (denoted by Z-F set theory); the characteristic feature of this theory is that the axiom of substitution consists of a countable number of statements, one for each definable relation $R(x, y)$, which say that if for some fixed set $A$ and for all $x$ in $A$ there exists a unique $y$ such that $R(x, y)$ holds, then there exists a set $B$ consisting of precisely those $y$. Since much of the proofs of the theorems we state follow quite closely the arguments of $[1]$, we shall be rather brief.

Our main result is

THEOREM 1. There exists a collection of sets which satisfy $Z-F$ set theory and such that any other such collection contains a sub-collection isomorphic to it.

Here, we mean that the $\in$-relation is taken to be the usual one. By

\footnotetext{
1 The author is a fellow of the Alfred P. Sloan Foundation.
} 
a well-founded model for set theory we mean one for which there does not exist a sequence $\left\{a_{n}\right\}, a_{n}$ in the model such that $a_{n+1} \in a_{n}$. A wellfounded model is always isomorphic to a model of actual sets. We may thus restate Theorem 1 by saying that there is a model for Z-F theory which is contained in every well-founded model. A collection $C$ of sets is called transitive if whenever $x \in C$ and $y \in x$ then $y \in C$. It is clear that every collection $C$ of sets is isomorphic (with respect to $\in$ ) to a transitive collection $C^{\prime}$. Namely, define $\phi$ by transfinite induction on the rank of $x$ for all $x \in C$, so that $\phi(x)=\{y \mid \exists z \in C, z \in x$, $\phi(z)=y\}$. With this remark, Theorem 1 follows from Theorem 3 below.

Let $T_{0}$ be any transitive collection of sets containing the set of all integers. For each ordinal $\alpha \geqq 1$ we define the set $T_{\alpha}$ by induction as follows:

Set $C_{\alpha}=\mathrm{U}_{\beta<\alpha} T_{\beta}$. Then:

(1) If $x$ and $y$ belong to $C_{\alpha},\{x, y\}$ belongs to $T_{\alpha}$.

(2) If $x \in C_{\alpha}, z=\{y \mid \exists u(y \in u \in x)\}$ belongs to $T_{\alpha}$.

(3) If $x \in C_{\alpha}, z=\left\{y \mid y \subseteq x, y \in C_{\alpha}\right\}$ belongs to $T_{\alpha}$.

(4) If $R(a, b)$ is any condition on sets $a, b$ constructed from the logical symbols and from sets in $C_{\alpha}$, and if for some fixed $x$ in $C_{\alpha}$, and all $y$ in $x$, there exists a unique $z$ in $C_{\alpha}$, such that $R(y, z)$ holds when all the quantifiers in $R$ are restricted to $C_{\alpha}$, then the set of all such $z \in T_{\alpha}$.

(5) Only the sets described in (1) through (4) belong to $T_{\alpha}$.

It is easy to see that $T_{\alpha}$ are an increasing sequence of sets.

We observe that this differs from Gödel's definition of constructible sets only in that we do not demand that $C_{\alpha}$ belong to $T_{\alpha}$.

The union $M$ of all $T_{\alpha}$ for all ordinals $\alpha$ we call the minimal model generated by $T_{0}$.

THEOREM 2. $M$ is a model for $Z-F$ set theory.

Proof. Precisely as in [1].

THEOREM 3. If $M^{\prime}$ is any transitive collection of sets such that $T_{0} \subseteq M^{\prime}$ for which $Z-F$ set theory holds, then $M^{\prime} \supseteq M$.

Proof. For each ordinal $\alpha$ in $M^{\prime}$, denote by $T_{\alpha}^{\prime}$ and $C_{\alpha}^{\prime}$ the sets formed within $M^{\prime}$ by precisely the same process as $T_{\alpha}$ and $C_{\alpha}$ are formed. It is clear that for $\alpha$ in $M^{\prime}, T_{\alpha}=T_{\alpha}^{\prime}$ and $C_{\alpha}=C_{\alpha}^{\prime}$. Let $M^{\prime \prime}$ denote the union of all $C_{\alpha}^{\prime}$ for $\alpha \in M^{\prime}$. Put

$$
\begin{aligned}
\Sigma & =\left\{\alpha \mid C_{\alpha+1} \neq C_{\alpha}\right\}, \\
\Sigma^{\prime} & =\left\{\alpha \mid \alpha \in M^{\prime}, C_{\alpha}^{\prime} \neq C_{\alpha+1}^{\prime}\right\} .
\end{aligned}
$$


Both $\Sigma$ and $\Sigma^{\prime}$ are clearly initial segments of ordinals. We assert that $\Sigma \subseteq \Sigma^{\prime}$. For, otherwise for some $\beta$ in $\Sigma, \beta$ is the supremum of $\Sigma^{\prime}$, and hence $C_{\beta}=M^{\prime \prime}$. Since $M^{\prime \prime}$ is a model for Z-F set theory it follows that operations (1) through (4) form no new sets when applied to $C_{\beta}$ and hence $C_{\beta+1}=C_{\beta}$, or $\beta \notin \Sigma$, a contradiction. Hence $T_{\alpha} \subseteq M^{\prime}$ for all $\alpha$ in $\Sigma$ and therefore $M^{\prime} \supseteq M$.

Theorem 4. If $T_{0}$ is countable, so is $M$.

Proof. By the Skolem-Löwenheim Theorem there is a countable model of sets containing $T_{0}$, so the theorem follows from Theorem 3.

Let $M_{0}$ denote the model $M$ where from now on $T_{0}$ is taken to be merely the integers and the set of all integers. $M_{0}$ is then the minimal model referred to, and is contained in every transitive model of sets for Z-F theory. It follows from the proof of Theorem 3 , that $\Sigma$ is contained in $M_{0}$ and thus that in $M_{0}$, the following axiom holds:

Strong Axiom of Constructibility. For every set $x$, there exists an ordinal $\alpha$ and $x \in T_{\alpha}$.

We note that there clearly are systems in which the strong Axiom of Constructibility fails, namely any uncountable model. It is also easy to see that the strong form implies the usual form of the Axiom of Constructibility.

Finally, we add an application to the problem of the independence of the Axiom of Choice.

THEOREM 5. Let $R(x)$ be any condition constructed from the logical symbols. Then using $Z-F$ set theory it is impossible to prove that the sets satisfying $R(x)$ form a model for $Z-F$ set theory and the negation of the Axiom of Choice (or even the negation of the Axiom of Constructibility).

Proof. If such a proof existed it would imply that the set of $x$ in $M_{0}$ satisfying $R(x)$ in $M_{0}$ would form such a model $N$. We may form the transitive model $N^{\prime}$, which is isomorphic to $N$ and which is still a submodel of $M_{0}$. Since $M_{0}$ is minimal, $N^{\prime}=M_{0}$ and so the Axiom of Constructibility holds in $N$. In Theorem 5 we allow the possibility that the proof that the axiom of substitution holds may involve a different proof for each one of the countable statements in that axiom.

Theorem 5 shows that in some sense the problem of independence of the Axiom of Choice is more difficult than that of the consistency, since in the latter case the relation $R(x)$ was taken to be that of constructible sets and the proof that in this collection of sets the Axiom of Choice held required only Z-F theory. Of course, Theorem 5 does not preclude the construction of such a model by means of operations 
going beyond Z-F theory, e.g. based on an enumeration of statements in Z-F theory and the truth or falsity thereof.

It is known [2] that no minimal model for analysis exists, i.e., a collection of sets of integers and satisfying the axioms of analysis which is contained in every other such collection. The difficulty would seem to be that there is no unique representation for ordinals in analysis corresponding to the standard representation in set theory. However, the above ideas may also be applied to analysis, and in this case one obtains the usual systems of ramified analysis, $A_{\alpha}$. Precisely as above one can show that their union is a model for analysis. Since the construction may be carried out within a countable model for set theory, it is clear, as before, that it is a countable model. The fact that the $A_{\alpha}$ stop at a countable ordinal can in fact be proved in Z-F theory. Observe that the $A_{\alpha}$ certainly stop for $\alpha=\omega_{1}$, the first uncountable ordinal. (If we take $\omega_{1}$ to be the first uncountable ordinal in $M_{0}$ we see immediately that the $A_{\alpha}$ definitely stop before the $C_{\alpha}$ do.) One can use the Skolem-Löwenheim argument to deduce that there is an increasing sequence of countable ordinals $\beta_{n}, \beta=\operatorname{Lim} \beta_{n}$, such that in $A_{\beta_{n}}$ and $A_{\beta}$ all the true statements are still true in $A_{\omega_{1}}$. From this it follows that any set of integers formed at $A_{\beta}$ is already identical to a set formed at $A_{\beta_{n}}$ for some $n$. Hence the process stops with $A_{\beta}$.

It remains open to find a model for analysis which is minimal in some natural class of models. In this direction Mostowski [3] has introduced the notion of a $\beta$-model, i.e., models in which the countable ordinals correspond to true well-orderings of the integers. It would be interesting to know whether in this class one can find a minimal model, and what the relation is with ramified analysis.

I wish to thank Professor Georg Kreisel and Professor Solomon Feferman for many stimulating conversations.

\section{REFERENCES}

1. K. Gödel, The consistency of the Continuum Hypothesis, Annals of Mathematics Studies No. 3, Princeton Univ. Press, Princeton, N. J., 1940.

2. R. V. Gandy, G. Kreisel and W. W. Tait, Set existence, Bull. Acad. Polon. Sci. 8 (1960), 577-582.

3. A. Mostowski, Formal system of analysis based on an infinitistic rule of proof, Infinitistic Methods, Proc. Sympos. Foundations Math., Warsaw, 1959, Pergamon Press, Oxford, 1961.

STANFORD UNIVERSITY 